\title{
Constructing effective field theories via their harmonics
}

\author{
Brian Henning ${ }^{1,2}$ and Tom Melia ${ }^{3}$ \\ ${ }^{1}$ Department of Physics, Yale University, New Haven, Connecticut 06511, USA \\ ${ }^{2}$ Départment de Physique Théorique, Université de Genève, 24 quai Ernest-Ansermet, \\ 1211 Genève 4, Switzerland \\ ${ }^{3}$ Kavli Institute for the Physics and Mathematics of the Universe (WPI), The University of Tokyo Institutes \\ for Advanced Study, The University of Tokyo, Kashiwa, Chiba 277-8583, Japan
}

(Received 25 March 2019; published 29 July 2019)

\begin{abstract}
We consider the construction of operator bases for massless, relativistic quantum field theories, and show this is equivalent to obtaining the harmonic modes of a physical manifold (the kinematic Grassmannian), upon which observables have support. This enables us to recast the approach of effective field theory (EFT) through the lens of harmonic analysis. We explicitly construct harmonics corresponding to low mass dimension EFT operators.
\end{abstract}

DOI: 10.1103/PhysRevD.100.016015

\section{INTRODUCTION}

The approach of effective field theory (EFT) is to consider all possible contributions to a given physical observable. Particle scatterings and decays only have support on a physical manifold where momentum conservation and on-shell conditions are satisfied. These constraints manifest as what are termed equations of motion (EOM) and integration by parts (IBP) relations between operators in the EFT, and have been the subject of extensive study spanning the past few decades [1-8].

In a series of papers [6-8], it was shown that these constraints are ultimately a consequence of the Poincaré symmetry of spacetime; this insight enabled a systematic enumeration of basis elements (i.e., operator counting) in an EFT. In particular, by considering a larger spacetime symmetry - that of the conformal group - it was shown the operator basis naturally consists of conformal primary operators, which could then be counted using Hilbert series techniques.

In this paper, we put operator construction on the same footing as operator enumeration, by detailing the systematic construction of the conformal primary operators that provide a privileged choice of basis for the $S$-matrix of the theory (for other approaches to operator basis construction, see $[5,6,8-12])$. The presentation is designed to accompany the paper [13], which considers more generally the entire operator spectrum (not just Lorentz scalars), as is relevant

Published by the American Physical Society under the terms of the Creative Commons Attribution 4.0 International license. Further distribution of this work must maintain attribution to the author(s) and the published article's title, journal citation, and DOI. Funded by SCOAP ${ }^{3}$. for more general correlation functions. This paper also proceeds more heuristically than [13] —in particular, by including a number of worked examples-and omits many mathematical details. We have endeavored to provide pointers to [13] in the relevant places. We would, however, like to refer the interested reader to [13] for a reinforced connection to ideas in conformal field theory (CFT), and modern (Hamiltonian truncation) nonperturbative methods.

We consider four dimensional relativistic theories of massless particles, and allow for all particle spins by working with spinor helicity variables, which encode both kinematic and helicity information. In these variables a $U(N)$ action on the phase space of $N$ particles is revealed, which generalizes the $U(1)^{N} \subset U(N)$ particle little group scalings. This symmetry plays a crucial role, first via a duality with the conformal group $S U(2,2) \simeq S O(4,2)$ that in [13] we term "conformal-helicity duality," and second via its symmetry breaking pattern which, in the case of EFTs, is down to $U(N-2) \times U(2)$, identifying the physical manifold as the Grassmann manifold $G_{2}\left(\mathbb{C}^{N}\right)=U(N) /(U(N-2) \times U(2))$.

A new picture of EFT emerges - that of harmonic analysis on the Grassmann manifold. There is a tight analogy with the harmonic analysis of a sphere: functions $f=f(x, y, z)$, with coordinates subject to the constraint $x^{2}+y^{2}+z^{2}=1$, can be expanded in terms of spherical harmonics on the sphere, $f=\sum_{l, m} c_{l m} Y_{l m}$. As we will see, in the EFT case the (Lorentz scalar) $N$-particle Hilbert space involving particles of any spin - subject to the constraints $p_{i}^{2}=0$ and $\sum_{i} p_{i}^{\mu}=P^{\mu}$-lives on the Grassmann manifold; it follows that the natural basis for the operators in the theory are the harmonics of this manifold.

For the case of the sphere, harmonic polynomials in $x, y$ and $z$ are annihilated by the Laplacian, $\nabla^{2}$; these form a basis of spherical harmonics when restricted to the sphere. 
For the EFT case, we will construct harmonic polynomials in spinor variables which are annihilated by a generalized Laplacian operator, $K$, that turns out to be the special conformal generator.

The paper has the following structure. In Sec. II we detail the construction of the EFT harmonics, presenting the main result from [13] and providing additional heuristic motivation. In Sec. III we use this result to explicitly construct low-lying harmonics thereby providing EFT bases at low mass dimension. Section IV concludes.

\section{CONSTRUCTING EFT HARMONICS}

In this section, we proceed to construct the operator basis, which follows from constructing operators which interpolate asymptotic states in the Hilbert space of the theory via the operator-state correspondence. Before detailing this construction, we remind the reader of the role of these states in the $S$-matrix, the calculation of which is the goal of EFT.

EFT quantifies all possible $S$-matrix elements between an $|i n\rangle$ state in a multiparticle Fock space and the vacuum,

$$
\langle 0|S| \text { in }\rangle .
$$

We consider massless asymptotic particle states ${ }^{1}$ labeled by kinematic (three momenta), helicity, and possibly some internal quantum numbers. Moreover, we consider multiparticle states that are built from distinguishable particles, deferring a discussion on exchange symmetry to Sec. IV.

We encode the kinematic information using spinor helicity variables,

$$
p_{i}^{\mu}\left(\bar{\sigma}^{\dot{a} a}\right)_{\mu}=\tilde{\lambda}_{i}^{\dot{a}} \lambda^{i a}, \quad \tilde{\lambda}_{i}^{\dot{a}}= \pm\left(\lambda^{i a}\right)^{*},
$$

with $a, \dot{a}=1,2$ the usual Lorentz indices and $i=1, \ldots, N$ a particle, or flavor, index [raised on $\lambda$ and lowered on $\tilde{\lambda}$ to anticipate the $U(N)$ action], and the \pm is for positive/ negative energies. Then $S$-matrix elements take the form

$$
\left\langle 0|S| \lambda^{1}, \tilde{\lambda}_{1}, h_{1} ; \ldots ; \lambda^{N}, \tilde{\lambda}_{N}, h_{N}\right\rangle=f\left(\left\{\lambda^{i}, \tilde{\lambda}_{i}\right\}\right) \delta^{(4)}\left(\sum_{i=1}^{N} \tilde{\lambda}_{i}^{\dot{a}} \lambda^{i a}\right),
$$

where $f\left(\left\{\lambda^{i}, \tilde{\lambda}_{i}\right\}\right)$ is a Lorentz scalar function of the spinor variables. In Eq. (3) we labeled states in the Fock space with spinors to encode the kinematic information, and with helicities $h_{i}$. In these variables, Lorentz invariant phase space is written as

$$
d \Phi_{N}=\prod_{i=1}^{N} d^{4} p_{i} \delta^{+}\left(p_{i}^{2}\right)=\prod_{i=1}^{N} \frac{d^{2} \lambda^{i} d^{2} \tilde{\lambda}_{i}}{\operatorname{Vol}(U(1))},
$$

\footnotetext{
${ }^{1}$ Massive states can be described via two massless states [up to an $S U(2)$ little group redundancy], see e.g., [14]; we will leave extensions in this direction to future work.
}

where $\delta^{+}\left(p_{i}^{2}\right)=\delta\left(p_{i}^{2}\right) \theta\left(p^{0}\right)$ and the volume of the little group $\operatorname{Vol}(U(1))=2 \pi$.

A typical way to think of EFT is as a theory defined by a Lagrangian containing a tower of operators; a trivial application of Dyson's formula shows that these interpolate the asymptotic states and lead to contact interactions at leading order in Dyson's formula. Said another way, the construction of an EFT basis, taking into account EOM and IBP, follows from the standard introduction of local operators as products of interpolating fields—see [8] for a detailed discussion on this point. Note that in using spinors, we automatically take into account the EOM (i.e., the momenta are on shell). The fields are required to transform under Poincaré in the way dictated by the helicity of the asymptotic state. For example, $\lambda^{a}$ transforms in the $\left(j_{1}, j_{2}\right)=\left(\frac{1}{2}, 0\right)$ representation (rep) and interpolates a negative helicity fermion $\psi_{L} ; \tilde{\lambda}^{\dot{a}} \tilde{\lambda}^{\dot{b}}$ transforms in the $(0,1)$ rep and interpolates a positive helicity massless spin-1 state [the field-strength operator $F_{R}=\frac{1}{2}(F+i \tilde{F})$ in spinor variables]; pairs of $\tilde{\lambda}^{a} \lambda^{a}$ imply a derivative acting on the interpolating field in the operator. In other words,

$$
F_{R}^{\dot{a} \dot{b}}=\int \frac{d^{2} \lambda d^{2} \tilde{\lambda}}{\operatorname{Vol}(U(1))}\left(\tilde{\lambda}^{\dot{a}} \tilde{\lambda}^{\dot{b}} e^{\frac{i}{2} \lambda^{a} \tilde{\lambda}^{a} x_{a \dot{a}}} a^{\dagger}+\text { H.c. }\right)
$$

etc. In this way, $f$ transforms under the asymptotic particle little groups with the correct helicity weight.

For the purpose of constructing a basis of operators for the EFT, it is natural to consider the kinematic configuration where all particles are incoming with positive energy, carrying total (nonzero) momentum $P$ [13]. This is because operators in a free field theory can be identified with the states in the Hilbert space of that theory via the stateoperator correspondence. States are formed by operators acting on the vacuum-: $\mathcal{O}(P):|0\rangle$, where $\mathcal{O}(P)$ is the Fourier transform of $\mathcal{O}(x)$ and the operator is normal ordered. $^{2}$ In $: \mathcal{O}(P):|0\rangle$ only the positive energy pieces contribute [e.g., the creation operator part in Eq. (5)]; for Lorentz scalar operators this results in a polynomial $f_{\mathcal{O}}(\lambda, \tilde{\lambda})$ constrained to the Grassmann manifold, $G_{2}\left(\mathbb{C}^{N}\right)$ (or for operators with spin, to the Stiefel manifold [13]). Note that the terms in $: \mathcal{O}(P)$ : involving annihilation operators can always be unambiguously recovered from the polynomial $f_{\mathcal{O}}$ by appropriate continuation of spinors from positive to negative energy. ${ }^{3}$

\footnotetext{
${ }^{2}$ The completeness of these states can be established for both free and interacting conformal field theories [15].

${ }^{3}$ Said another way, operators $\mathcal{O}(x)$ in free theories are polynomials in fields and their derivatives from which-upon plugging in mode decompositions for the fields like in Eq. (5)—one finds polynomials $f_{\mathcal{O}}(\lambda, \tilde{\lambda})$. We are only stating the unsurprising fact that this map is invertible; given $f_{\mathcal{O}}(\lambda, \tilde{\lambda})$, one can unambiguously construct the associated position space polynomial in fields and their derivatives.
} 
To make the above explicit, we proceed to analyze the geometry. We first write the spinors as

$$
\left(\begin{array}{llll}
\lambda^{11} & \lambda^{21} & \cdots & \lambda^{N 1} \\
\lambda^{12} & \lambda^{22} & \cdots & \lambda^{N 2}
\end{array}\right)=\left(\begin{array}{l}
\mathbf{u} \\
\mathbf{v}
\end{array}\right)
$$

In this way one sees that the vectors $\mathbf{u}$ and $\mathbf{v}$ define a 2-plane; under Lorentz transformations $\mathbf{u}$ and $\mathbf{v}$ rotate within the plane so that, modulo these transformations, Lorentz invariant phase space is described as the set of 2-planes that intersect the origin in $\mathbb{C}^{N}$, which defines $G_{2}\left(\mathbb{C}^{N}\right)$. A more general manifold is obtained if one does not mod out by Lorentz rotations [13] - this case is most easily analyzed by considering the breaking of the $U(N)$ symmetry (under which $\mathbf{u}$ and $\mathbf{v}$ transform as fundamentals) down to $U(N-2)$ from the constraint that the spinors carry net momentum $P$,

$$
P^{\dot{a} a}=\left(\begin{array}{cc}
|\mathbf{u}|^{2} & \mathbf{u}^{\dagger} \cdot \mathbf{v} \\
\mathbf{v}^{\dagger} \cdot \mathbf{u} & |\mathbf{v}|^{2}
\end{array}\right) .
$$

The manifold that the coset $U(N) / U(N-2)$ defines is known as the Stiefel manifold.

Working in the center of mass frame, $P^{\dot{a} a}=M \operatorname{diag}(1,1)$, and using the $U(N)$ action, under which Eq. (7) is invariant, to set

$$
\left(\begin{array}{l}
\mathbf{u} \\
\mathbf{v}
\end{array}\right)=\sqrt{M}\left(\begin{array}{ccccc}
1 & 0 & 0 & \cdots & 0 \\
0 & 1 & 0 & \cdots & 0
\end{array}\right)
$$

The remaining $U(N-2)$ freedom is manifest in this frame, acting on the $N-2$ columns of zeros in the above equation. Let us now consider the $U(2) \simeq S U(2) \times U(1)$ that acts on the first two columns of the matrix on the rhs. The diagonal combination of this $S U(2)$ together with the $S U(2) \subset$ $S L(2, \mathbb{C})$ enacting Lorentz rotations further preserves the above matrix; therefore, for Lorentz invariant states, it can be further factored out. The remaining $U(1)$ is an overall phase $(\mathbf{u}, \mathbf{v})^{T} \rightarrow e^{i \alpha}(\mathbf{u}, \mathbf{v})^{T}$; for cases when the state has zero total helicity [see below, Eq. (14)], this too can be factored out; the resulting geometry is identified with the coset $U(N) /(U(N-2) \times U(2))$, which is the Grassmann manifold. ${ }^{4}$

Returning to the analogy with the sphere where the Laplacian $\nabla^{2}$ in essence forms an adjoint to $|\mathbf{r}|^{2}$, we construct the adjoint operator to $P^{\dot{a} a}$ as

\footnotetext{
${ }^{4}$ For the case of nonzero total helicity, states transform under this phase, and the resulting Grassmann manifold is $S U(N) / S(U(N-2) \times U(2))$. This total helicity is interesting in that it is the diagonal $U(1)$ in the $U(N) \simeq S U(N) \times U(1)$ and could equally have been associated to the conformal group $U(2,2) \simeq S U(2,2) \times U(1)$ [13]. While there remain physical ramifications to fully understand in these points, we nevertheless stress that the algebraic construction of operators elucidated in this work is blind to these nuanced details.
}

$$
K_{\dot{a} a}=-\sum_{i=1}^{N} \frac{\partial}{\partial \tilde{\lambda}_{i}^{\dot{a}}} \frac{\partial}{\partial \lambda^{i a}}
$$

which is the generator of special conformal transformations in spinor variables. The harmonic modes of the Grassmann manifold are those annihilated by $K$; they are thus identified with primary conformal operators, and we therefore turn to constructing such harmonic polynomials.

\section{A. Harmonics from Young diagrams}

Let us build basis polynomials out of $n \lambda \mathrm{s}$ and $\tilde{n} \tilde{\lambda}_{\mathrm{s}}$, at fixed $N \geq 2$. Because the polynomials are Lorentz scalars, $n$ and $\tilde{n}$ must be even, with the spinors contracted as

$$
\left[j_{1} j_{2}\right] \ldots\left[j_{\tilde{n}-1} j_{\tilde{n}}\right]\left\langle i_{1} i_{2}\right\rangle \ldots\left\langle i_{n-1} i_{n}\right\rangle,
$$

where we use bracket notation $\langle i j\rangle=\lambda^{i a} \lambda_{a}^{j},[i j]=\tilde{\lambda}_{i a} \tilde{\lambda}_{j}^{\dot{a}}$, and where the indices $i_{1} \ldots i_{n}, j_{1} \ldots j_{\tilde{n}}$ are (unspecified as yet) particle indices.

We consider raised particle number indices on $\lambda$ as $U(N)$ indices, such that $\lambda^{i a}$ transforms under $S L(2, \mathbb{C}) \times U(N)$ as spinor $\times$ fundamental. Similarly, $\tilde{\lambda}_{i}^{\dot{a}}$ transforms as (the complex conjugate representation) spinor $\times$ antifundamental. That is, the indices $i_{1}$ to $i_{n}$ in Eq. (10) can be interpreted as (raised) $U(N)$ indices, and the indices $j_{1}$ to $j_{\tilde{n}}$ can be interpreted as (lowered) conjugate $U(N)$ indices. The latter can be raised using the epsilon tensor,

$\left[j_{1} j_{2}\right] \epsilon^{j_{1} j_{2} k_{1} \ldots k_{N-2}} \ldots\left[j_{\tilde{n}-1} j_{\tilde{n}}\right] \epsilon^{j_{\tilde{n}-1} j_{\tilde{n}} l_{1} \ldots l_{N-2}}\left\langle i_{1} i_{2}\right\rangle \ldots\left\langle i_{n-1} i_{n}\right\rangle$,

with summation over all $j$ indices.

The key result of [13] is that the basis polynomials furnish a particular representation of $U(N)$, labeled by eigenvalues $n$ and $\tilde{n}$. Equivalently one can label by mass dimension $\Delta$, and helicity $h{ }^{5}$

$$
\begin{gathered}
\Delta=\frac{1}{2}(n+\tilde{n})+N, \\
h=\frac{1}{2}(n-\tilde{n}) .
\end{gathered}
$$

Finite dimensional representations of $U(N)$ are in oneto-one correspondence with Young diagrams-see e.g., [16]. That is, the Young diagrams encode the symmetrization pattern to be applied to the indices in Eq. (11), to form a $U(N)$ irreducible representation. The particular Young diagram that renders Eq. (11) a harmonic mode of the

\footnotetext{
${ }^{5}$ To provide a translation to the notation used in [13], here $n=l_{1}+l_{2}$ and $\tilde{n}=\tilde{l}_{1}+\tilde{l}_{2}$ in the Lorentz scalar case where $l_{1}=l_{2}$ and $\tilde{l}_{1}=\tilde{l}_{2}$. We note that more general non-Lorentzscalar operators are further labeled by spin eigenvalues, $j_{1}$ and $j_{2}$.
} 


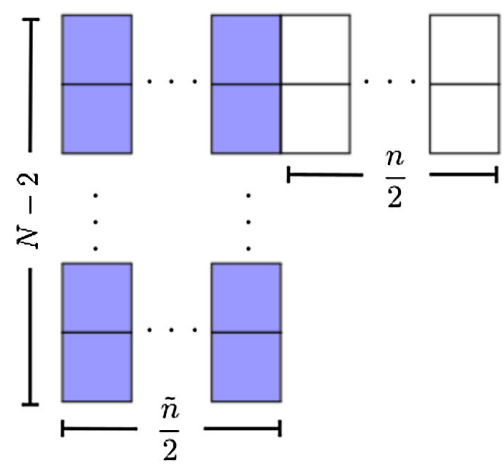

FIG. 1. Young diagram for the harmonic modes of the Grassmannian.

Grassmannian is given in Fig. 1. The indices $k_{1} \ldots k_{N-2}$ in Eq. (11) are associated with the first column which is shaded blue (to indicate it corresponds to $\tilde{\lambda}$ indices raised with an epsilon tensor); the indices $l_{1} \ldots l_{N-2}$ in Eq. (11) are associated with the rightmost blue column; the indices $i_{1}, i_{2}$ with the leftmost unshaded column; and, the indices $i_{n-1}, i_{n}$ with the final column:

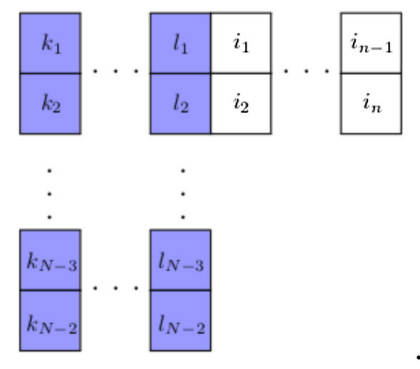

A basis for the $U(N)$ rep is supplied by semistandard Young tableaux, as discussed in the next subsection. For now, we want to reflect upon why it is that this representation is primary.

To begin to understand this result, let us start by considering holomorphic operators-that is, functions consisting purely of $\lambda \mathrm{s}$. These are obviously primary (annihilated by $K$ ). We consider basis functions that are polynomials in a fixed number $n$ of $\lambda$ s. These $\lambda$ carry two indices, $\lambda^{i a}$. A simple but important observation is that if a symmetrization pattern is applied to one index, the other index automatically inherits this pattern. For example,

$$
\lambda^{i a} \lambda^{j b}+(i \leftrightarrow j)=\lambda^{i a} \lambda^{j b}+\lambda^{j a} \lambda^{i b},
$$

is a symmetrization in particle indices $i$ and $j$, but the resulting expression is also symmetric in $a$ and $b$. Similarly,

$$
\lambda^{i a} \lambda^{j b}-(i \leftrightarrow j)=\lambda^{i a} \lambda^{j b}-\lambda^{j a} \lambda^{i b},
$$

antisymmetrizes in $i$ and $j$; the antisymmetry is inherited by $a$ and $b$ as well. This works for general symmetrization patterns that are encoded by the Young diagrams. So, when a polynomial in $n \lambda \mathrm{s}$ is organized into a singlet representation of $S L(2, \mathbb{C})$ - corresponding to a Young diagram with $n / 2$ boxes in the first row and $n / 2$ boxes in the second row-the $U(N)$ indices inherit the exact same symmetrization pattern,

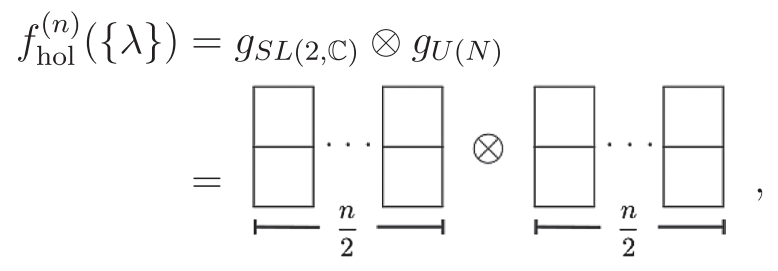

Note that this implies that $U(N)$ representations corresponding to Young diagrams with more than two rows-i.e., that are antisymmetrized on more than two indices - can never be constructed, e.g., $\lambda^{i a} \lambda^{j b} \lambda^{k c}+($ anti-sym in $i, j, k)=0$, for all $a, b, c$.

The above considerations apply to antiholomorphic basis functions in $\tilde{n} \tilde{\lambda}$ s: again, the $U(N)$ representation is dictated by the symmetrization pattern on the Lorentz indices such that the functions are Lorentz scalars,

$$
\begin{aligned}
& f_{\text {anti-hol }}^{(\widetilde{n})}(\{\widetilde{\lambda}\})=g_{S L(2, \mathbb{C})}^{\star} \otimes \bar{g}_{U(N)} \\
& =\square_{\frac{n}{2}} \ldots
\end{aligned}
$$
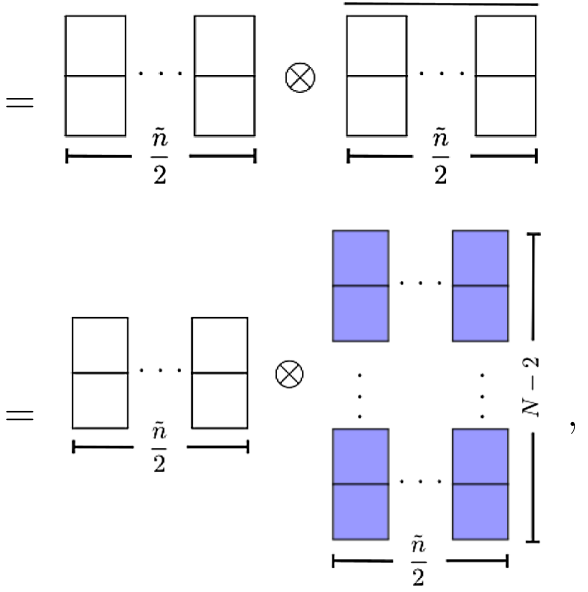

where we used a barred Young diagram to denote the conjugate $U(N)$ representation; in the last equality we redrew this as the $\epsilon$ tensor conjugated diagram.

Now we turn to the nonholomorphic case, concerning $n$ $\lambda$ s and $\tilde{n} \tilde{\lambda}_{\text {s. Such operators only appear for } N \geq 4 \text {, which }}$ reflects the familiar fact that Mandelstam invariants are trivial for $N \leq 3$ [13]. The $\lambda$ s and $\tilde{\lambda}_{\text {s separately have their }}$ $S L(2, \mathbb{C})$ indices symmetrized into the Lorentz scalar patterns as in the holomorphic and antiholomorphic cases above; again the $U(N)$ indices and conjugate $U(N)$ indices will inherit the same pattern. What is different this time, is that now the resulting $U(N)$ representation is reducible, 


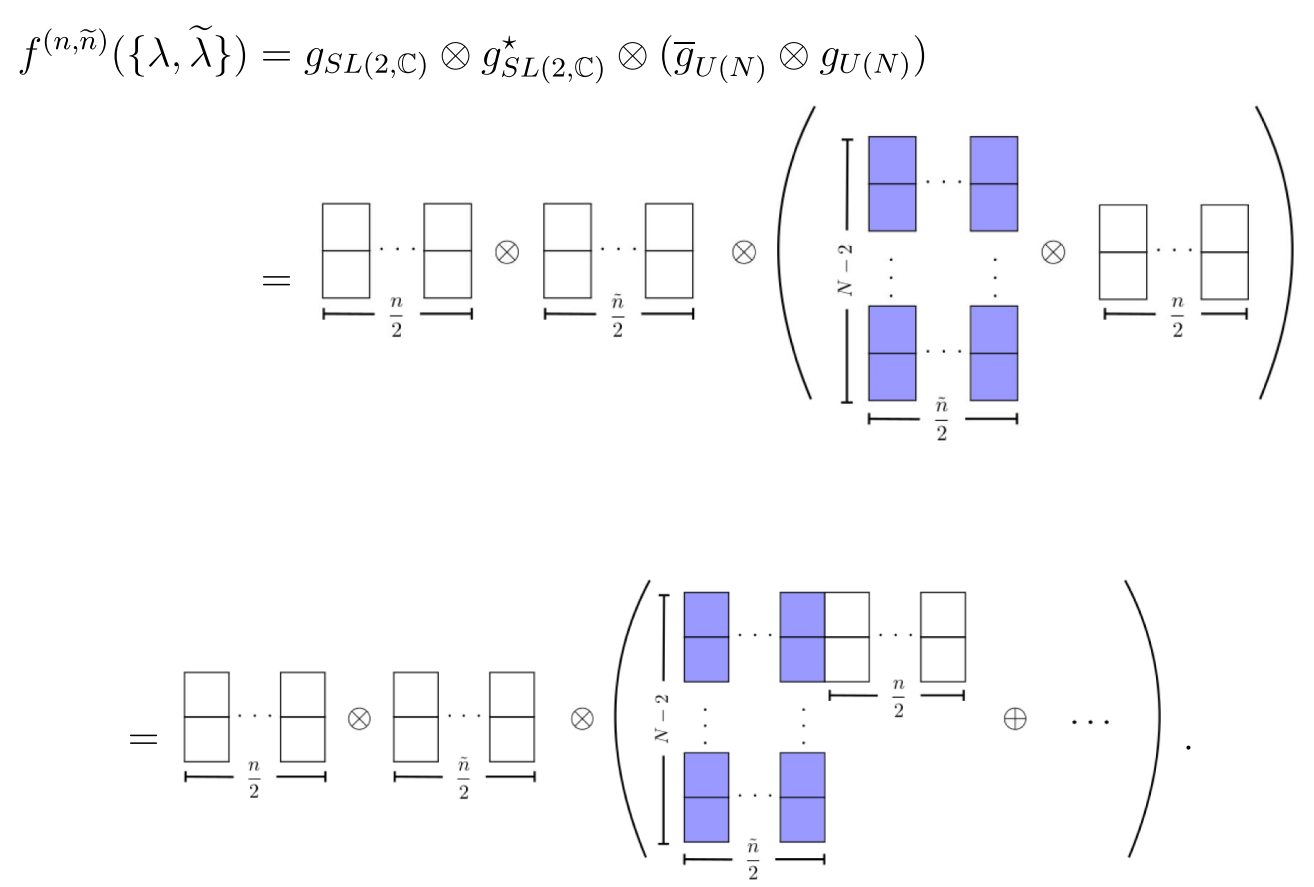

In the last equality, the $U(N)$ tensor decomposition is indicated, displaying only the leading term; this term coincides with the Young diagram in Fig. 1 and renders the polynomial harmonic, which we prove at the end of this section. This term is leading in the sense that it is the only $U(N)$ representation in the decomposition that does not contain an overall factor of momentum, $P$, and thus the only primary operator/ harmonic mode in the decomposition. We now turn to proving this.

The familiar diagrammatic "box placing" rules for carrying out tensor decompositions with Young diagrams (Littlewood-Richardson rules, again, see e.g., [16]) can be applied to the product in Eq. (18). The leading term appearing in Eq. (19) is in fact the simplest representation obtained using these rules - no white boxes have been shifted around, and the Young tableaux have been simply stuck together.

What of the other "..." terms in Eq. (19)? The box placing rules specify that we end up with a Young diagram that has either one or two white boxes at the bottom of a blue box column of length $N-2$. For the case of one white box under a column of $N-2$ blue boxes, we can factor a term in the resulting diagram that has the form

$$
\left[j_{1} j_{2}\right] \epsilon^{j_{1} j_{2} k_{1} . k_{N-2}}\left\langle i_{1}\right|+\left(\operatorname{anti}-\operatorname{sym} \operatorname{in} k_{1}, \ldots, k_{N-2}, i_{1}\right) .
$$

By the antisymmetry, the indices $k_{1}, \ldots, k_{N-2}, i_{1}$ must be distinct choices of $1 \ldots N$ (otherwise the antisymmetrization sets this factor to zero); without loss of generality, we consider the choice $1, \ldots, N-1$. Each cyclicly related set of terms in the above anti-sym is proportional (by a sign) to

$$
\sum_{k=1}^{N-1}[N k]\langle k|=[N \mid P-[N N]\langle N|=[N \mid P,
$$

using $\left.P=\sum_{k=1}^{N} \mid k\right]\langle k|$ and $[N N]=0$. Equation (20), as promised, contains a factor of total momentum, $P$, and thus the operator is a descendant.

For the case of two white boxes under a column of $N-2$ blue boxes, one proceeds similarly: first, we factor a term

$$
\left[j_{1} j_{2}\right] \epsilon^{j_{1} j_{2} k_{1} . . k_{N-2}}\left\langle i_{1}\right|\left\langle i_{2}\right|+\left(\text { anti-sym in } k_{1}, \ldots, k_{N-2}, i_{1}, i_{2}\right) .
$$

(The spinors $\left\langle i_{1}\right|$ and $\left\langle i_{2}\right|$ could be contracted, $\left\langle i_{1} i_{2}\right\rangle$; the below arguments are valid in this case too.) The indices $k_{1}, \ldots, k_{N-2}, i_{1}, i_{2}$ are antisymmetrized permutations of the set $1 \ldots N$. Evidently, for any fixed value of $i_{2}$, one can factor out $P$ as per Eq. (20); in fact, one can easily show that in summing over the other values of $i_{2}$, a factor of $P^{2}$ can be pulled out overall.

This shows that the additional $U(N)$ representations are descendants, because they have the overall factor of $P$. We will return to a proof that the leading Young diagram Eq. (19) is annihilated by $K$ very shortly, showing that it is primary, after the introduction of semistandard Young tableaux.

\section{B. States from semistandard Young tableaux}

For a given Young diagram, one can construct the states of the corresponding $U(N)$ representation using semistandard Young tableau (SSYT), which we will see provides the labeling of the little group scaling. We recall that a SSYT is a filling of the boxes of a Young diagram 
with the numbers 1 through $N$ (repeated use of a number is allowed) subject to the following rules:

(i) The numbers along the rows must weakly increase (i.e., reading from left to right each subsequent number must be greater than or equal to the previous one).

(ii) The numbers down the columns must strongly increase (i.e., reading from top to bottom each
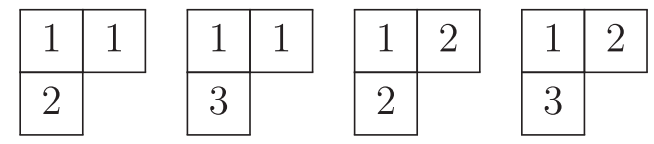

For a given SSYT of the Young diagram in Fig. 1, one easily constructs the basis polynomial in $\lambda$ and $\tilde{\lambda}$ using the diagram symmetrization rules (sym on rows, anti-sym on columns). It is then straightforward to read off the field content by the little group scaling for each particle; equivalently these are the eigenvalues of the $U(1)^{N} \subset$ $U(N)$ generators. Note that the little group scaling of pairs of $\lambda^{i}, \tilde{\lambda}_{i}$ cancel; for each such pair one should count a derivative to the field content of the harmonic/operator; i.e., $\lambda_{a}^{i} \tilde{\lambda}_{i \dot{a}}=p_{a \dot{a}}^{i}$ is the momentum of the $i$ th particle (a derivative acting on the field for the $i$ th particle). While each term in the polynomial must scale the same way under the little group overall, the pairs of $\lambda^{i}, \tilde{\lambda}_{i}$ could appear (and do appear) for different particle numbers $i$ in different terms.

We point out that the SSYT fillings will separately construct harmonics for all possible spins of each external state. For example, harmonics corresponding to each of the operators $F_{L 1} F_{L 2} \phi_{3}, F_{L 1} \phi_{2} F_{L_{3}}$, and $\phi_{1} F_{L 2} F_{L 3}$ will be included separately. However, it is clear that these operators are of exactly the same form and can be related to each other with a simple particle index permutation. We emphasize we are dealing with all-distinguishable particles, and that such a permutation is between particle species; it is not the (anti)symmetrization necessary when to describe indistinguishable particles. We can define a set of reduced SSYT which mods out such permutations between particle species with a simple ordering rule:

order on SSYT filling: $1 \mathrm{~s} \geq 2 \mathrm{~s} \geq \cdots \geq N \mathrm{~s}$.

That this is true is proven in the Appendix.

As promised, we now return to the proof that all states of the representation shown in Fig. 1 are annihilated by $K=-\sum \partial \tilde{\partial}$. Consider the highest weight state, corresponding to the filling of all the boxes in the first row with 1 s, all those in the second row with 2 s, etc. Such a state is trivially annihilated by $K$ : it consists only of polynomials in the four variables $\lambda_{1}, \lambda_{2}, \tilde{\lambda}_{N-1}$ and $\tilde{\lambda}_{N}$. The rest of the proof follows by group theory: since $K$ is a $U(N)$ singlet, its action commutes with the action of the $U(N)$ raising and subsequent number must be greater than the previous one).

The number of valid SSYT is equal to the dimension of the $U(N)$ representation. For example, for the eightdimensional adjoint representation of $U(3)$ we find eight SSYT fillings:

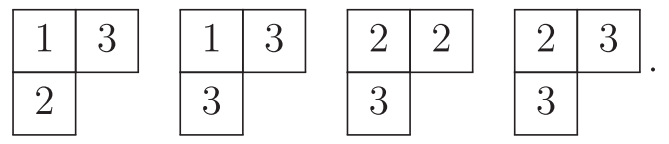

lowering operators, and as such annihilates all the states in the representation.

We conclude this section with a discussion on the orthogonality of the harmonics constructed via the Young tableaux of Fig. 1, under the phase space measure of Eq. (4). ${ }^{6}$ First, operators at different $N$ are orthogonal due to the Fock space structure of the Hilbert space. Given the $U(N)$ symmetry of phase space, it is also clear that $U(N)$ representations with different $n, \tilde{n}$ are automatically orthogonal. What of the states within each representation? The integral over the little group for each individual particle ensures that states with different eigenvalues of the torus $U(1)^{N}$ are automatically orthogonal as well. In general, however, there exist degenerate subspaces where more than one operator has equal little group eigenvalues (the SSYT are permutations of each other). In such cases, state orthogonality is not guaranteed; we postpone discussion of this point (and details of normalization with respect to the phase space volume) to a future detailed, systematic study of the harmonics.

\section{EFT SPECTRA AT LOW MASS DIMENSION}

It is instructive to work through the construction of harmonics/operators at low values of $n$ and $\tilde{n}$, i.e., at low mass dimension, $\Delta$. In the following, we work through examples that suffice to construct an EFT basis up to mass dimension six.

The formalism above provides a recipe to perform the construction:

(1) Write down the Young diagram corresponding to the choice of $n$ and $\tilde{n}$, as shown in Fig. 1.

(2) Write down all semistandard Young tableau (SSYT) fillings to construct the $U(N)$ states. $^{7}$

The operators we construct are summarized in Tables I-III.

\footnotetext{
${ }^{6}$ For an explicit formulation of phase space in terms of Grassmannian variables, see [17].

${ }^{7}$ Alternatively, any other method of constructing the states could be used, e.g., start with the highest weight state and apply lowering operators.
} 
TABLE I. Reduced SSYT for Lorentz scalar operators of the form $(n, \tilde{n})=(2,0),(0,2)$ for all $N \geq 3$.

\begin{tabular}{cc}
\hline \hline & $\begin{array}{c}1 \\
2 \\
1\end{array}$ \\
\hline 2 & $\cdot$ \\
& $\cdot$ \\
$(2,0)$ & $(0,2)$ \\
\hline$\phi^{N-2} \psi_{L}^{2}$ & $\phi^{N-2} \psi_{R}^{2}$ \\
\hline \hline
\end{tabular}

We will highlight the special features of this conformal basis as we come across them. Of particular importance is the structure of the harmonics when annihilation by $K$ is nontrivial. Such a case happens when the corresponding operator involves derivatives, which is also where IBP relations come into play; these operators are necessarily nonholomorphic. Another feature is the grouping of harmonics/operators with differing field content as states of the same $U(N)$ representation.

Below we normalize the Young tableaux permutations with a factor $1 / k$,

$$
k=\prod_{i \in \text { rows }} \prod_{j \in \text { columns }} p_{i} ! q_{j} !,
$$

where $p_{i}$ is the number of boxes in the $i$ th row, and $q_{j}$ is the number of boxes in the $j$ th column of the tableaux.

\section{A. Harmonics of type $(n, \tilde{n})=(2,0),(0,2)$}

We begin with harmonics for which $(n, \tilde{n})=(2,0)$, $(0,2)$. These are the simplest (nontrivial) harmonics, and
TABLE II. Reduced SSYT for Lorentz scalar operators with $N=3$, for low values of $n, \tilde{n}$. $\xi$ denotes a spin $3 / 2$ field.

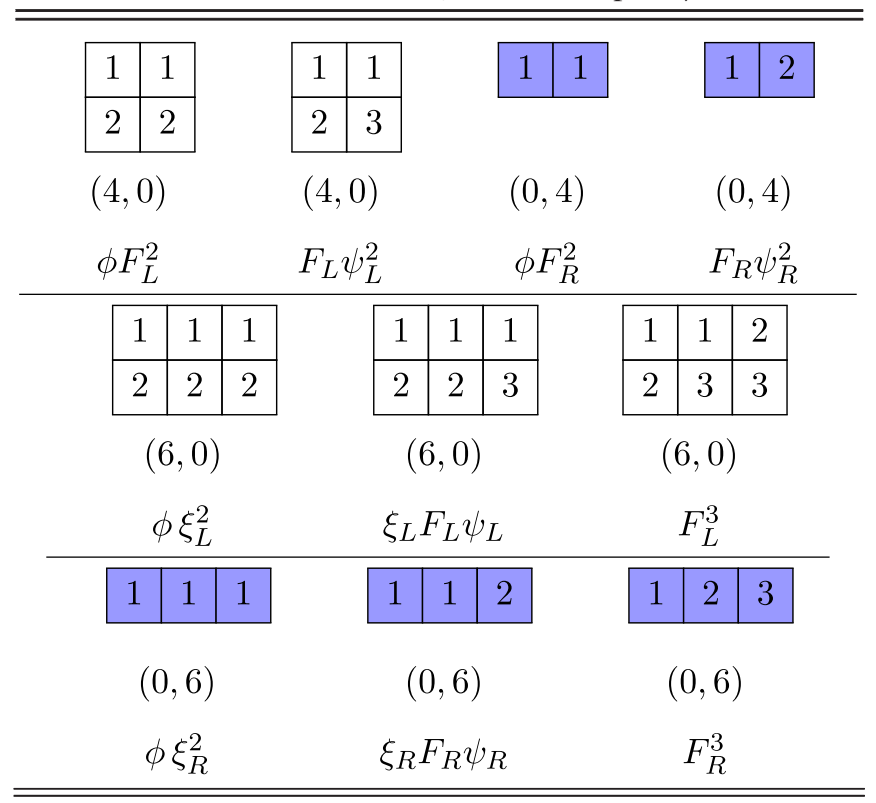

we consider them for all $N \geq 2$. The relevant reduced SSYT are displayed in Table I. They correspond to operators of field content $\phi^{N-2} \psi_{L}^{2}$ and $\phi^{N-2} \psi_{R}^{2}$, respectively. We reemphasize that we consider distinguishable particles at this point; the particle index is suppressed in the Table, but we indicate it explicitly in the following construction:

$$
\phi_{3} \ldots \phi_{N} \psi_{L 1} \psi_{L 2}
$$

$\phi_{1} \ldots \phi_{N-2} \psi_{R N-1} \psi_{R N}:$

$$
\begin{aligned}
& \begin{array}{l}
1 \\
\hline 2 \\
\cdot
\end{array}=\frac{1}{(N-2) !} \widetilde{\lambda}_{j_{1} \dot{a}} \widetilde{\lambda}_{j_{2}}^{\dot{a}}\left(\epsilon^{j_{1} j_{2} 1 . . N-2}+\text { anti-sym. in } 1 . . N-2\right), \\
& \cdot \cdot N-2 \\
& =[N-1 N],
\end{aligned}
$$


TABLE III. Reduced SSYT for Lorentz scalar operators with $N=4$, for low values of $n, \tilde{n}$.

\begin{tabular}{|c|c|c|c|c|}
\hline & & $(4,0)$ & $(2,2)$ & $(0,4)$ \\
\hline 1 & 1 & \multirow{2}{*}{$\phi^{2} F_{L}^{2}$} & \multirow{2}{*}{$\psi_{L}^{2} \psi_{R}^{2}$} & \multirow{2}{*}{$\phi^{2} F_{R}^{2}$} \\
\hline 2 & 2 & & & \\
\hline 1 & 1 & \multirow{2}{*}{$\phi F_{L} \psi_{L}^{2}$} & \multirow{2}{*}{$\phi^{2} \psi_{L} \psi_{R} D$} & \multirow{2}{*}{$\phi F_{R} \psi_{R}^{2}$} \\
\hline 2 & 3 & & & \\
\hline 1 & 2 & \multirow{2}{*}{$\psi_{L}^{4}$} & \multirow[t]{2}{*}{$\phi^{4} D^{2}$} & \multirow{2}{*}{$\psi_{R}^{4}$} \\
\hline 3 & 4 & & & \\
\hline 1 & 3 & \multirow{2}{*}{$\psi_{L}^{4}$} & \multirow{2}{*}{$\phi^{4} D^{2}$} & \multirow{2}{*}{$\psi_{R}^{4}$} \\
\hline 2 & 4 & & & \\
\hline
\end{tabular}

where in eq. (23a) summation over $j_{1}$ and $j_{2}$ is implied. Putting back in the flavor permutations, there are $N(N-1) / 2$ SSYT obtained from each of the reduced ones in Eqs. (22) and (23b). Note that the operators are conjugate to each other, i.e., $L \leftrightarrow R$ in all fields, and are thus related by switching $\lambda \leftrightarrow \tilde{\lambda}$, or \langle\rangle$\leftrightarrow[]$.

\section{B. Low "frequency" harmonics for $N=3$}

Next, we fix the number of particles in the harmonic to be $N=3$, and consider harmonics of low $n$ and $\tilde{n}$. The case $N=3$ is special, as the construction given in Fig. 1 does not produce a valid Young tableau when both $n$ and $\tilde{n}$ are nonzero. This reflects the fact that all of the Lorentz scalar harmonics/operators for $N=3$ are holomorphic (or antiholomorphic). In Table II we consider the cases $(n, \tilde{n})=(4,0),(0,4),(6,0),(0,6)$, and show the reduced SSYT. Note how harmonics with different field content are grouped into the same $U(N)$ representation; for example the harmonics $\phi F_{L}^{2}$ and $F_{L} \psi_{L}^{2}$ both appear as states in the $(4,0)$ representation.

The left-handed holomorphic $N=3$ operators in Table II are constructed as follows:

$\phi_{3} F_{L 1} F_{L 2}:$

$$
\begin{array}{rl}
\hline 1 & 1 \\
\hline 2 & 2 \\
\hline & =\frac{1}{(2 !)^{4}}\left(\lambda^{1 a} \lambda_{a}^{2} \lambda^{1 b} \lambda_{b}^{2}+\text { tab. perms }\right), \\
& =\frac{2^{2}}{(2 !)^{4}}\left(\lambda^{1 a} \lambda_{a}^{2} \lambda^{1 b} \lambda_{b}^{2}-\lambda^{2 a} \lambda_{a}^{1} \lambda^{1 b} \lambda_{b}^{2}-\lambda^{1 a} \lambda_{a}^{2} \lambda^{2 b} \lambda_{b}^{1}+\lambda^{2 a} \lambda_{a}^{1} \lambda^{2 b} \lambda_{b}^{1}\right) \\
& =\langle 12\rangle^{2} .
\end{array}
$$

$F_{L 1} \psi_{L 2} \psi_{L 3}:$

$$
\begin{aligned}
& \begin{array}{|l|l|}
\hline 1 & 1 \\
\hline 2 & 3
\end{array}=\frac{2^{2}}{(2 !)^{4}}\left(\lambda^{1 a} \lambda_{a}^{2} \lambda^{1 b} \lambda_{b}^{3}-\lambda^{2 a} \lambda_{a}^{1} \lambda^{1 b} \lambda_{b}^{3}-\lambda^{1 a} \lambda_{a}^{2} \lambda^{3 b} \lambda_{b}^{1}+\lambda^{2 a} \lambda_{a}^{1} \lambda^{3 b} \lambda_{b}^{1}\right), \\
& =\langle 12\rangle\langle 13\rangle \text {. }
\end{aligned}
$$

$\phi_{3} \xi_{L 1} \xi_{L 2}$

$$
\begin{aligned}
& \begin{array}{|l|l|l|}
\hline 1 & 1 & 1 \\
\hline 2 & 2 & 2 \\
\hline
\end{array}=\frac{1}{(3 !)^{2}(2 !)^{3}}\left(\lambda^{1 a} \lambda_{a}^{2} \lambda^{1 b} \lambda_{b}^{2} \lambda^{1 c} \lambda_{c}^{2}+\text { tab. perms }\right), \\
& =\langle 12\rangle^{3} \text {. }
\end{aligned}
$$




$$
\xi_{L 1} F_{L 2} \psi_{L 3}:
$$

$$
\begin{aligned}
& \begin{array}{|l|l|l|}
\hline 1 & 1 & 1 \\
\hline 2 & 2 & 3
\end{array}=\frac{1}{(3 !)^{2}(2 !)^{3}}\left(\lambda^{1 a} \lambda_{a}^{2} \lambda^{1 b} \lambda_{b}^{2} \lambda^{1 c} \lambda_{c}^{3}+\text { tab. perms }\right), \\
& =\langle 12\rangle^{2}\langle 13\rangle \text {. }
\end{aligned}
$$

$F_{L 1} F_{L 2} F_{L 3}:$

$$
\begin{aligned}
& \begin{array}{|l|l|l|}
\hline 1 & 1 & 2 \\
\hline 2 & 3 & 3 \\
\hline
\end{array}=\frac{1}{(3 !)^{2}(2 !)^{3}}\left(\lambda^{1 a} \lambda_{a}^{2} \lambda^{1 b} \lambda_{b}^{3} \lambda^{2 c} \lambda_{c}^{3}+\text { tab. perms }\right), \\
& =\langle 12\rangle\langle 13\rangle\langle 23\rangle \text {. }
\end{aligned}
$$

In the above we made use of the formula $\lambda^{i a} \lambda_{a}^{j}=-\lambda_{a}^{i} \lambda^{j a}$. The remaining right-handed holomorphic operators in Table II can be obtained (up to flavor permutations) by exchanging $\lambda \rightarrow \tilde{\lambda}$, i.e., $\langle i j\rangle \rightarrow[i j]$. However, we work out one case from the tableaux explicitly, for illustrative purposes,

$$
\psi_{R 1} \psi_{R 2} F_{R 3}:
$$

$$
\begin{array}{rl}
\hline 1 & 2 \\
\hline & =\frac{1}{2 !} \widetilde{\lambda}_{j_{1} \dot{a}} \widetilde{\lambda}_{j_{2}}^{\dot{a}} \widetilde{\lambda}_{j_{3} \dot{b}} \widetilde{\lambda}_{j_{4}}^{b}\left(\epsilon^{j_{1} j_{2} 1} \epsilon^{j_{3} j_{4} 2}+\epsilon^{j_{1} j_{2} 2} \epsilon^{j_{3} j_{4} 1}\right), \\
& =[13][32],
\end{array}
$$

where in the above summation over $j_{1}, j_{2}, j_{3}, j_{4}$ is implied. This is indeed the conjugate of (a flavor permutation of) Eq. (25).

\section{Low "frequency" harmonics for $N=4$}

As a last class of examples, we consider harmonics involving $N=4$ fields; reduced $\operatorname{SSYT}$ for $(n, \tilde{n})=$ $(4,0),(2,2),(0,4)$ are shown in Table III. There are two new features evident in the Table that were not present for the cases considered above. The first feature is that now nonholomorphic harmonics appear (the middle column). We will comment on the detailed form of these operators below. The second feature is that there are distinct harmonics with the same field content: two copies of the harmonic $\psi_{L}^{4} / \psi_{R}^{4}$ appear in the $(4,0) /(0,4) U(N)$ representation, and two copies of $\phi^{4} D^{2}$ appear in the $(2,2)$ representation. These operators are independent, so it is important that they are both included; the rules for constructing the reduced SSYT ensure this happens.

The left-handed holomorphic ones are constructed as follows [the first two are identical to the operators in Eqs. (24) and (25), respectively, differing only by the addition of an extra $\phi$ field]:

$$
\phi_{3} \phi_{4} F_{L 1} F_{L 2}:
$$$$
\begin{array}{|l|l|}
\hline 1 & 1 \\
\hline 2 & 2 \\
\hline
\end{array}=\langle 12\rangle^{2} .
$$

$$
\begin{aligned}
& \phi_{4} F_{L 1} \psi_{L 2} \psi_{L 3}: \\
& \qquad \begin{array}{|l|l|}
\hline 1 & 1 \\
\hline 2 & 3 \\
\hline
\end{array}=\langle 12\rangle\langle 13\rangle . \\
& \psi_{L 1} \psi_{L 2} \psi_{L 3} \psi_{L 4}: \\
& \begin{array}{|l|l|}
\hline 1 & 2 \\
\hline 3 & 4
\end{array}=\frac{1}{(2 !)^{4}}\left(\lambda^{1 a} \lambda_{a}^{3} \lambda^{2 b} \lambda_{b}^{4}+\text { tab. perms }\right), \\
& =\frac{1}{2}(\langle 14\rangle\langle 23\rangle+\langle 13\rangle\langle 24\rangle) .
\end{aligned}
$$


$\psi_{L 1} \psi_{L 2} \psi_{L 3} \psi_{L 4}:$

$$
\begin{array}{rl}
\hline 1 & 3 \\
\hline 2 & 4 \\
\hline n= & =\frac{1}{(2 !)^{4}}\left(\lambda^{1 a} \lambda_{a}^{2} \lambda^{3 b} \lambda_{b}^{4}+\text { tab. perms }\right), \\
& =\frac{1}{2}(-\langle 14\rangle\langle 23\rangle+\langle 12\rangle\langle 34\rangle) .
\end{array}
$$

The right-handed holomorphic harmonics in Table III are obtained via conjugation of Eqs. (30)-(33), and so we do not present their construction explicitly.

Turning finally to the nonholomorphic harmonics, we have

$\psi_{L 1} \psi_{L 2} \psi_{R 3} \psi_{R 4}:$

$$
\begin{aligned}
& \begin{array}{|l|l|}
\hline 1 & 1 \\
\hline 2 & 2 \\
\hline
\end{array}=\frac{1}{(2 !)^{4}} \widetilde{\lambda}_{j_{1} \dot{a}} \widetilde{\lambda}_{j_{2}}^{\dot{a}}\left(\epsilon^{j_{1} j_{2} 12} \lambda^{1 a} \lambda_{a}^{2}+\text { tab. perms }\right), \\
& =\frac{2^{2}}{(2 !)^{4}} \widetilde{\lambda}_{j_{1} \dot{a}} \widetilde{\lambda}_{j_{2}}^{\dot{a}}\left(\epsilon^{j_{1} j_{2} 12} \lambda^{1 a} \lambda_{a}^{2}-\epsilon^{j_{1} j_{2} 21} \lambda^{1 a} \lambda_{a}^{2}-\epsilon^{j_{1} j_{2} 12} \lambda^{2 a} \lambda_{a}^{1}+\epsilon^{j_{1} j_{2} 21} \lambda^{2 a} \lambda_{a}^{1}\right), \\
& =\langle 12\rangle[34] \text {. }
\end{aligned}
$$

$\phi_{2} \phi_{3} \psi_{L 1} \psi_{R 4} D:$

$$
\begin{aligned}
& \begin{array}{|l|l|}
\hline 1 & 1 \\
\hline 2 & 3 \\
\hline
\end{array}=\frac{1}{(2 !)^{4}} \widetilde{\lambda}_{j_{1} \dot{a}} \widetilde{\lambda}_{j_{2}}^{\dot{a}}\left(\epsilon^{j_{1} j_{2} 12} \lambda^{1 a} \lambda_{a}^{3}+\text { tab. perms }\right), \\
& =\frac{2}{(2 !)^{4}} \widetilde{\lambda}_{j_{1} \dot{a}} \widetilde{\lambda}_{j_{2}}^{\dot{a}}\left(\epsilon^{j_{1} j_{2} 12} \lambda^{1 a} \lambda_{a}^{3}+\epsilon^{j_{1} j_{2} 13} \lambda^{1 a} \lambda_{a}^{2}+\text { tab. anti-syms }\right), \\
& =\frac{1}{2}(\langle 13\rangle[34]-\langle 12\rangle[24]) \text {. }
\end{aligned}
$$

$\phi_{1} \phi_{2} \phi_{3} \phi_{4} D^{2}$ :

$$
\begin{array}{rl}
\hline 1 & 2 \\
\hline 3 & 4 \\
= & \frac{1}{(2 !)^{4}} \widetilde{\lambda}_{j_{1} \dot{a}} \widetilde{\lambda}_{j_{2}}^{\dot{a}}\left(\epsilon^{j_{1} j_{2} 13} \lambda^{2 a} \lambda_{a}^{4}+\text { tab. perms }\right) \\
= & \frac{1}{(2 !)^{4}} \widetilde{\lambda}_{j_{1} \dot{a}} \widetilde{\lambda}_{j_{2}}^{\dot{a}}\left(\epsilon^{j_{1} j_{2} 13} \lambda^{2 a} \lambda_{a}^{4}+\epsilon^{j_{1} j_{2} 23} \lambda^{1 a} \lambda_{a}^{4}\right. \\
& \left.\quad+\epsilon^{j_{1} j_{2} 14} \lambda^{2 a} \lambda_{a}^{3}+\epsilon^{j_{1} j_{2} 24} \lambda^{1 a} \lambda_{a}^{3}+\text { tab. anti-syms }\right), \\
= & \frac{1}{4}(\langle 24\rangle[42]-\langle 14\rangle[41]-\langle 23\rangle[32]+\langle 13\rangle[31]) .
\end{array}
$$




$$
\phi_{1} \phi_{2} \phi_{3} \phi_{4} D^{2}:
$$

$$
\begin{aligned}
& \begin{array}{|l|l|}
\hline 1 & 3 \\
\hline 2 & 4 \\
\hline
\end{array}=\frac{1}{(2 !)^{4}} \widetilde{\lambda}_{j_{1}} \widetilde{\lambda}_{j_{2}}^{\dot{a}}\left(\epsilon^{j_{1} j_{2} 12} \lambda^{3 a} \lambda_{a}^{4}+\text { tab. perms }\right), \\
& =\frac{1}{4}(\langle 34\rangle[43]-\langle 14\rangle[41]-\langle 23\rangle[32]+\langle 12\rangle[21]) \text {. }
\end{aligned}
$$

The last three of these have nontrivial annihilation by $K$. For example, the harmonic in Eq. (35) with operator content $\phi^{2} \psi_{L} \psi_{R} D$,

$$
\begin{aligned}
& \sum_{i=1}^{N} \frac{\partial}{\partial \tilde{\lambda}_{i}^{\dot{a}}} \frac{\partial}{\partial \lambda^{i a}}\left(\frac{1}{2}(\langle 13\rangle[34]-\langle 12\rangle[24])\right) \\
& \quad=\frac{1}{2}\left(\lambda^{1 a} \tilde{\lambda}_{4}^{\dot{a}}-\lambda^{1 a} \tilde{\lambda}_{4}^{\dot{a}}\right)=0 .
\end{aligned}
$$

Using momentum conservation, one could rewrite the operator Eq. (35) as another equally valid operator basis element, e.g., simply $\langle 13\rangle[34]$ or $\langle 12\rangle[24]$, but it is only the combination $\propto(\langle 13\rangle[34]-\langle 12\rangle[24])$ that is a conformal primary and is annihilated by $K$ as in Eq. (38); it is in this sense that the harmonics form a privileged basis.

\section{DISCUSSION}

The general construction above applies to the distinguishable particles case. To take into account exchange symmetry one must (anti)symmetrize over the identical (fermionic) bosonic fields in an operator. The particle index can also be interpreted as a gauge or other symmetry index; further bookkeeping is required here too. The kinematic construction detailed here is a necessary first step (and the above considerations can be easily applied by hand, if not entirely systematically at present).

To the EFTer, the systematic nature of the construction is clearly appealing. The automatic orthogonality of (the majority of operators) at different $N$ and with different $U(1)^{N}$ eigenvalue of basis elements also has utility: converting from a UV Lagrangian/other EFT parametrization is then simple, via a projection $\int d \Phi_{N} Y^{*} \mathcal{L}_{\text {other }}$. It will be useful to further study orthogonality in the degenerate eigenvalue case. It would also be interesting to explore how this "mathematically singled out" basis fares in phenomenological applications.

There is deep structure in the operator basis which should be explored further. One of the interesting features is the mixing of different particle species within the same harmonic (e.g., the columns in Table III)—does this imply any relation between different phenomenological observables? We note that these harmonic blocks are the same grouping as the classes in the nonrenormalization theorems [18-20], and may shed further light on the structure of EFT anomalous dimension matrices/amplitude noninterference
[21] results. Of further interest is whether the harmonic picture presented here sheds further light or provides tools for studying positivity-type constraints on Wilson coefficients [22-26]; it would also be interesting to understand the connection between this natural basis and natural bases for amplitudes, e.g., partial waves.

\section{ACKNOWLEDGMENTS}

We thank Peter Cox, Marc Riembau, and Francesco Riva for conversations, and Rodrigo Alonso and Peter Cox for comments on the draft. B.H. is funded by the Swiss National Science Foundation under Grant No. PP002170578. T.M. is supported by the World Premier International Research Center Initiative (WPI), MEXT, Japan, and by JSPS KAKENHI Grant No. JP18K13533.

\section{APPENDIX: REDUCED TABLEAUX}

When operators are related by simple index permutations between particle species that do not change the form of the operator, e.g., $F_{L 1} F_{L 2} \phi_{3}, F_{L 1} \phi_{2} F_{L_{3}}$, and $\phi_{1} F_{L 2} F_{L 3}$, we wish to define a rule so as to only consider one of them. A canonical choice is to keep only operators in which the fields are helicity ordered, such that those of lower helicity are assigned lower particle indices. In the example above, this would be the operator $F_{L 1} F_{L 2} \phi_{3}$. (Right-handed fields have positive helicity, so if we replace all instances of $L \rightarrow R$ in the above example, the canonical choice would be $\phi_{1} F_{R 2} F_{R 3}$.)

More precisely, an operator is not of this canonical form if the following is true: there exists a pair of fields in the operator that has particle index $i$ and $j$ with $i<j$, but has helicities satisfying $h_{i}>h_{j}$. After removing such operators, we call the remaining set reduced operators. We will show that the SSYT corresponding to a reduced operator satisfies

order on SSYT filling: $1 \mathrm{~s} \geq 2 \mathrm{~s} \geq \cdots \geq N \mathrm{~s}$.

Before turning to the proof, note that if $h_{i}=h_{j}$ there is no notion of a canonical order on $i$ or $j$ in defining a reduced operator. That is, the set of reduced operators includes operators related by nontrivial permutations of the indices between fields of equal helicity. For illustration, we take two examples from the text. First, consider the SSYT for $N=3$, 


$$
\begin{array}{|l|l|}
\hline 1 & 1 \\
\hline 2 & 3 \\
\hline
\end{array} \quad: \quad F_{L 1} \psi_{L 2} \psi_{L 3} \text {. }
$$

The corresponding operator has a single field of helicity -1 and two fields of helicity $-\frac{1}{2}$, and it is of reduced form. There are no nontrivial index permutations between the two fermions. [The Young tableaux corresponding to this permutation is not semistandard - it would be the filling $(1,1)$, $(3,2)$.] However, there are index permutations between the sets of fields to create the operators $F_{L 2} \psi_{L 1} \psi_{L 3}$ and $F_{L 3} \psi_{L 1} \psi_{L 2}$, which correspond to SSYT fillings $(1,2)$, $(2,3)$ and $(1,2),(3,3)$. These two operators are not reduced, and are discarded by the ordering rule above; indeed, the SSYT fillings do not satisfy Eq. (A1).

For the second example consider the SSYTs for $N=4$,

\begin{tabular}{|l|l|}
\hline 1 & 2 \\
\hline 3 & 4 \\
\hline
\end{tabular} and \begin{tabular}{|c|c|}
\hline 1 & 3 \\
\hline 2 & 4 \\
\hline
\end{tabular}

both of which are operators with field content $\psi_{L 1} \psi_{L 2} \psi_{L 3} \psi_{L 4}$ and are related by nontrivial particle index permutations between particles of equal helicity. Both are included in the reduced set of operators. ${ }^{8}$

\footnotetext{
${ }^{8}$ This highlights an issue with how the particle index permutations are implemented across the set of reduced operators. To illustrate this, denote the two operators from the example (A3) as $\left(\psi_{L 1} \psi_{L 2} \psi_{L 3} \psi_{L 4}\right)^{A}$ and $\left(\psi_{L 1} \psi_{L 2} \psi_{L 3} \psi_{L 4}\right)^{B}$. Now consider two reduced operators that exist in the $N=7$ ring: $\left(\psi_{L 1} \psi_{L 2} \psi_{L 3} \psi_{L 4}\right)^{A} \phi_{5} \phi_{6} \phi_{7}$ and $\left(\psi_{L 1} \psi_{L 2} \psi_{L 3} \psi_{L 4}\right)^{B} \phi_{5} \phi_{6} \phi_{7}$. These are related by index permutations to the nonreduced operators $\left(\psi_{L 1} \psi_{L 5} \psi_{L 6} \psi_{L 7}\right)^{A} \phi_{2} \phi_{3} \phi_{4}$ and $\left(\psi_{L 1} \psi_{L 5} \psi_{L 6} \psi_{L 7}\right)^{B} \phi_{2} \phi_{3} \phi_{4}$. It would be incorrect to perform the permutation to the two operators differently, such that one could obtain e.g., $\left(\psi_{L 1} \psi_{L 5} \psi_{L 6} \psi_{L 7}\right)^{A} \phi_{2} \phi_{3} \phi_{4}$ and $\left(\psi_{L 1} \psi_{L 6} \psi_{L 5} \psi_{L 7}\right)^{B} \phi_{2} \phi_{3} \phi_{4}$, which are in fact identical operators.
}

We now turn to proving that the statement on SSYT in Eq. (A1) follows for an operator that is of reduced form. First, consider the holomorphic case. Here, each field of helicity $h_{i}$ necessitates $2\left|h_{i}\right|$ copies of $\lambda^{i}$ in the operator, which in turn necessitates $2\left|h_{i}\right|$ copies of the box $i$ in the SSYT filling. Since for a reduced operator $\left|h_{1}\right| \geq\left|h_{2}\right| \geq \cdots \geq\left|h_{N}\right|$, and all helicities $h_{i} \leq 0$ in the holomorphic case, the condition Eq. (A1) immediately follows.

Next, consider the antiholomorphic case, where all the helicities $h_{i} \geq 0$. A field of helicity $h_{i}$ necessitates $2 h_{i}$ copies of $\tilde{\lambda}_{i}$ in the operator. Each Lorentz contracted pair of $\tilde{\lambda}_{i \dot{a}} \tilde{\lambda}_{j}^{\dot{a}}$ necessitates a column in the SSYT of $N-2$ blue boxes filled with the numbers 1 to $N$, excluding $i$ and $j$. Since $h_{N} \geq h_{N-1} \geq \cdots \geq h_{1}$, the number $N$ will be excluded in the SSYT more (or equal) times than the number $N-1$, which in turn will be excluded more (or equal) times than $N-2$, etc., and again the condition Eq. (A1) follows.

For the nonholomorphic case, first let us assume that no derivatives are present in the operator. In this case, we split the particles into negative helicity (to which we apply the same reasoning in the holomorphic case) and into positive helicity (to which we apply the nonholomorphic reasoning), and conclude again that the condition Eq. (A1) holds.

Finally we need to show that derivatives do not change the counting. A derivative implies a pair $\tilde{\lambda}_{i} \lambda^{i}$ (no sum on $i$ ) in the operator. It is useful to consider the $\tilde{\lambda}_{i}$ as contributing $N-1$ boxes to the SSYT filled with the numbers 1 to $N$, excluding $i$; when it is contracted with a $\tilde{\lambda}_{j}$, a box $j$ is further removed. The $\lambda^{i}$ in the pair contributes a (white) box $i$. Thus we see that the contribution of $\tilde{\lambda}_{i} \lambda^{i}$ to the SSYT filling is to add a set of $N$ boxes that contains one copy each of the numbers 1 to $N$. As such, it does not affect the condition Eq. (A1).
[1] W. Buchmuller and D. Wyler, Effective Lagrangian analysis of new interactions and flavor conservation, Nucl. Phys. B268, 621 (1986).

[2] B. Grzadkowski, M. Iskrzynski, M. Misiak, and J. Rosiek, Dimension-six terms in the Standard Model Lagrangian, J. High Energy Phys. 10 (2010) 085.

[3] E. E. Jenkins, A. V. Manohar, and M. Trott, Renormalization group evolution of the Standard Model dimension six operators I: Formalism and lambda dependence, J. High Energy Phys. 10 (2013) 087.

[4] L. Lehman, Extending the Standard Model effective field theory with the complete set of dimension-7 operators, Phys. Rev. D 90, 125023 (2014).
[5] L. Lehman and A. Martin, Hilbert series for constructing Lagrangians: Expanding the phenomenologist's toolbox, Phys. Rev. D 91, 105014 (2015).

[6] B. Henning, X. Lu, T. Melia, and H. Murayama, Hilbert series and operator bases with derivatives in effective field theories, Commun. Math. Phys. 347, 363 (2016).

[7] B. Henning, X. Lu, T. Melia, and H. Murayama, 2, 84, 30, 993, 560, 15456, 11962, 261485, ...: Higher dimension operators in the SM EFT, J. High Energy Phys. 08 (2017) 016.

[8] B. Henning, X. Lu, T. Melia, and H. Murayama, Operator bases, $S$-matrices, and their partition functions, J. High Energy Phys. 10 (2017) 199. 
[9] L. Lehman and A. Martin, Low-derivative operators of the Standard Model effective field theory via Hilbert series methods, J. High Energy Phys. 02 (2016) 081.

[10] B. Gripaios and D. Sutherland, DEFT: A program for operators in EFT, J. High Energy Phys. 01 (2019) 128.

[11] Y. Shadmi and Y. Weiss, Effective field theory amplitudes the on-shell way: Scalar and vector couplings to gluons, J. High Energy Phys. 02 (2019) 165.

[12] J. C. Criado, BasisGen: Automatic generation of operator bases, Eur. Phys. J. C 79, 256 (2019).

[13] B. Henning and T. Melia, Conformal-helicity duality and the Hilbert space of free CFTs, arXiv:1902.06747.

[14] N. Arkani-Hamed, T.-C. Huang, and Y.-t. Huang, Scattering amplitudes for all masses and spins, arXiv:1709.04891.

[15] M. Gillioz, X. Lu, and M. A. Luty, Scale anomalies, states, and rates in conformal field theory, J. High Energy Phys. 04 (2017) 171.

[16] H. Georgi, Lie algebras in particle physics. From isospin to unified theories, Front. Phys. 54, 1 (1982).

[17] P. Cox and T. Melia, Independently parametrized momenta variables and Monte Carlo IR subtraction, J. High Energy Phys. 12 (2018) 038.

[18] R. Alonso, E. E. Jenkins, and A. V. Manohar, Holomorphy without supersymmetry in the Standard Model effective field theory, Phys. Lett. B 739, 95 (2014).
[19] C. Cheung and C.-H. Shen, Nonrenormalization Theorems without Supersymmetry, Phys. Rev. Lett. 115, 071601 (2015).

[20] J. Elias-Miro, J. R. Espinosa, and A. Pomarol, One-loop nonrenormalization results in EFTs, Phys. Lett. B 747, 272 (2015).

[21] A. Azatov, R. Contino, C. S. Machado, and F. Riva, Helicity selection rules and noninterference for BSM amplitudes, Phys. Rev. D 95, 065014 (2017).

[22] M. Froissart, Asymptotic behavior and subtractions in the Mandelstam representation, Phys. Rev. 123, 1053 (1961).

[23] V. N. Gribov, Possible asymptotic behavior of elastic scattering, JETP Lett. 41, 667 (1961).

[24] A. Adams, N. Arkani-Hamed, S. Dubovsky, A. Nicolis, and R. Rattazzi, Causality, analyticity and an IR obstruction to UV completion, J. High Energy Phys. 10 (2006) 014.

[25] B. Bellazzini, F. Riva, J. Serra, and F. Sgarlata, Beyond Positivity Bounds and the Fate of Massive Gravity, Phys. Rev. Lett. 120, 161101 (2018).

[26] C. de Rham, S. Melville, A. J. Tolley, and S.-Y. Zhou, UV complete me: Positivity bounds for particles with spin, J. High Energy Phys. 03 (2018) 011. 